\title{
Application of single-cell Hi-C method for analysis of lampbrush chromosome conformation
}

\author{
Nurislamov A. ${ }^{1,2 *}$, Gridina M. ${ }^{2}$, Taskina A. ${ }^{1}$, Fishman V. ${ }^{1,2}$ \\ ${ }^{1}$ Novosibirsk State University, Novosibirsk, Russia \\ ${ }^{2}$ Institute of Cytology and Genetics SB RAS, Novosibirsk, Russia \\ *e-mail: a.nurislamov@g.nsu.ru
}

Key words: Hi-C, single-cell, lampbrush chromosomes, oogenesis

Motivation and Aim: Despite being discovered in late 19th century, lampbrush chromosomes' structure is still not well studied: most of the lateral loops have not yet been mapped and the study of their threedimensional organization by microscopic methods does not allow precise identifying of molecular mechanisms involved in their formation $[3,4]$. The rapid improvement of methods for capturing the spatial conformation of chromosomes (3C) in recent years has significantly expanded the range of their application and also made it possible to conduct a deeper analysis at the level of individual cells [1]. Such single-cell techniques allow to reveal heterogeneity in chromatin conformation within populations of the same cell type [2] and require less input material to work with. Here, we aimed to adopt single-cell Hi-C method for studying chromatin interactions in a developing oocyte nucleus.

Methods and Algorithms: Nuclei were extracted from chicken oocytes as described in [3]. Hi-C libraries were prepared by using modified version of protocol shown in [2]. Libraries were sequenced by Illumina. NGS data was analyzed to construct Hi-C maps.

Results: As shown by microscopic methods, fixation of nuclei preserves specific spatial organization of lampbrush chromatin, which indicates that $\mathrm{Hi}-\mathrm{C}$ could be used to infer native chromatin structure. Analysis of NGS data in some samples revealed the absence of whole chromosomes and the presence of a large number of reads that do not align with the chicken genome. In samples where these issues weren't present, two types of Hi-C maps were visually noticed - with a high and low proportion of trans-contacts. For most of the analyzed nuclei we observed a noticeable drop of the contact frequencies for loci separated by more than $1-2 \mathrm{Mb}$.

Conclusion: Experimental data show that the single-cell Hi-C method can be applied to analyze the conformation of lampbrush chromosomes. Further analysis requires optimization of protocol workflow and bioinformatics algorithms.

Acknowledgements: This project was supported by the RSF grant 20-64-46021.

\section{References}

1. Nagano T. et al. Single-cell Hi-C for genome-wide detection of chromatin interactions that occur simultaneously in a single cell. Nature Protocols. 2015;10(12):1986.

2. Nagano T. et al. Single-cell Hi-C reveals cell-to-cell variability in chromosome structure. Nature. 2013;502(7469):5964.

3. Zlotina A. et al. Microdissection of lampbrush chromosomes as an approach for generation of locus-specific FISH-probes and samples for high-throughput sequencing. BMC Genomics. 2016:17(1):126.

4. Zlotina A. et al. New insights into chromosome organization provided by lampbrush chromosome microdissection and high-throughput sequencing. Frontiers Genetics. 2020;11:57. 